\title{
Bursitis por mercurio tras accidente laboral: a propósito de un caso
}

\section{Bursitis due to Mercury after a work accident: \\ a case report}

\section{Susana Lastras González}

Servicio de Prevención de Riesgos Laborales. Hospital Ramón y Cajal. Madrid. España.

\section{Ignacio Sánchez-Arcilla}

Servicio de Prevención de Riesgos Laborales. Hospital Ramón y Cajal. Madrid. España.

\section{Marina Fernández Escribano}

Servicio de Prevención de Riesgos Laborales. Hospital Ramón y Cajal. Madrid. España.

\section{Magdalena Muedra Sánchez}

Servicio de Prevención de Riesgos Laborales. Hospital Ramón y Cajal. Madrid. España.

Recibido: 12-04-10

Aceptado: 28-05-10

\section{Correspondencia:}

Susana Lastras González

Balcánica 3, bloque C, 3. D

28042 Madrid. España

Tfno: 620586301

e-mail: sulasg $1 @$ @hotmail.com

\section{Resumen}

Auxiliar de enfermería de 28 años que acude al Servicio de Urgencias porque refiere dolor y dificultad para la movilización en rodilla derecha, tras accidente laboral, hace dos días. Refiere que se resbaló mientras transportaba 32 termómetros de mercurio, rompiéndose éstos sobre su rodilla, creando una pequeña herida, siendo la puerta de entrada del metal. En la radiografía, se objetiva el cuerpo extraño metálico, correspondiente a mercurio, en bursa rotuliana. Tras el diagnóstico, se realizó la extracción de la bursa, recuperándose la trabajadora totalmente tras la intervención.

Con este caso clínico que aportamos, queremos conocer los efectos del mercurio en la salud de las personas expuestas a dicho metal, estudiar los errores acontecidos en este accidente laboral, valorando cuáles hubieran sido las medidas de actuación preventiva adecuadas para evitar la repetición de este tipo de accidentes y, por último, reflexionar acerca de la relación beneficio-riesgo del uso del mercurio en aparatos de medición en el ámbito sanitario.

Med Segur Trab (Internet) 2010; 56 (219): 158-164

Palabras clave: accidente laboral, bursitis rotuliana, mercurio. 


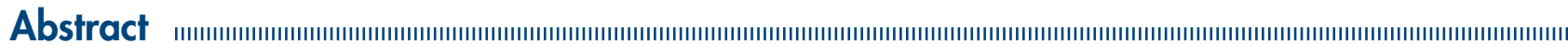

A 28-year-old woman, nursing assistant, who attends to emergency service, referring pain and difficulty mobilizing right knee after a work accident two days ago. She relates that slipped while carrying 32 mercury thermometers, breaking them on her knee, creating a small wound, being the gateway to the metal. In the radiograph, we objective a metallic foreign body, corresponding to mercury, in patellar bursa. After the diagnosis, the bursa was extracted, and the worker recovered completely, after surgery.

With this case report we bring, we want to know the health effects of mercury in people exposed to this metal, studying the errors occurred in this accident, assessing measures of preventive action that would have been neccesary to preclude recurrence of this type accidents and, finally, think about the risk-benefit balance the use of mercury in measuring devices in healthcare.

Med Segur Trab (Internet) 2010; 56 (219): 158-164

Keywords: work accident, patellar bursitis, mercury. 


\section{CASO CLÍNICO}

Mujer de 28 años, auxiliar de enfermería, que acude al Servicio de Traumatología de Urgencias porque refiere que desde hace dos días comienza con dolor en la región subpatelar de la rodilla derecha que ha ido empeorando y actualmente, tiene dificultad para la movilización de dicha articulación.

En la exploración física, se objetiva una lesión eritematosa en región subpatelar derecha, con dolor a la palpación y una pequeña herida inciso-contusa de escasos milímetros de longitud. La movilización activa y pasiva están limitadas parcialmente por el dolor, no presentando ningún otro síntoma acompañante.

La trabajadora refiere que en la infancia tuvo un osteocondroma en la misma rodilla, actualmente curado y sin secuelas, realizándose la última radiografía de control hace dos meses, siendo ésta completamente normal.

La trabajadora relaciona este episodio con un accidente laboral sufrido hace dos días, en el que mientras transportaba en la mano una caja con 32 termómetros de mercurio, se resbaló y cayó al suelo. Algunos de los termómetros de mercurio cayeron sobre su rodilla derecha y le produjeron una herida inciso-contusa, que observamos aún en la exploración en estado de cicatrización.

La auxiliar de enfermería reconoce que tenía sobrecarga en su puesto de trabajo. La caja en la que transportaba los termómetros no estaba cerrada, la trabajadora no llevaba guantes y la ropa de trabajo no fue retirada tras el accidente laboral. Posteriormente, no se realizó ninguna medida higiénico-sanitaria ni se actuó de forma adecuada frente al derrame de mercurio.

En el servicio de Urgencias, el siguiente paso fue la realización de una radiografía de la rodilla, objetivándose un cuerpo extraño metálico en bursa rotuliana, correspondiente al mercurio. (Figuras 1 y 2 ).

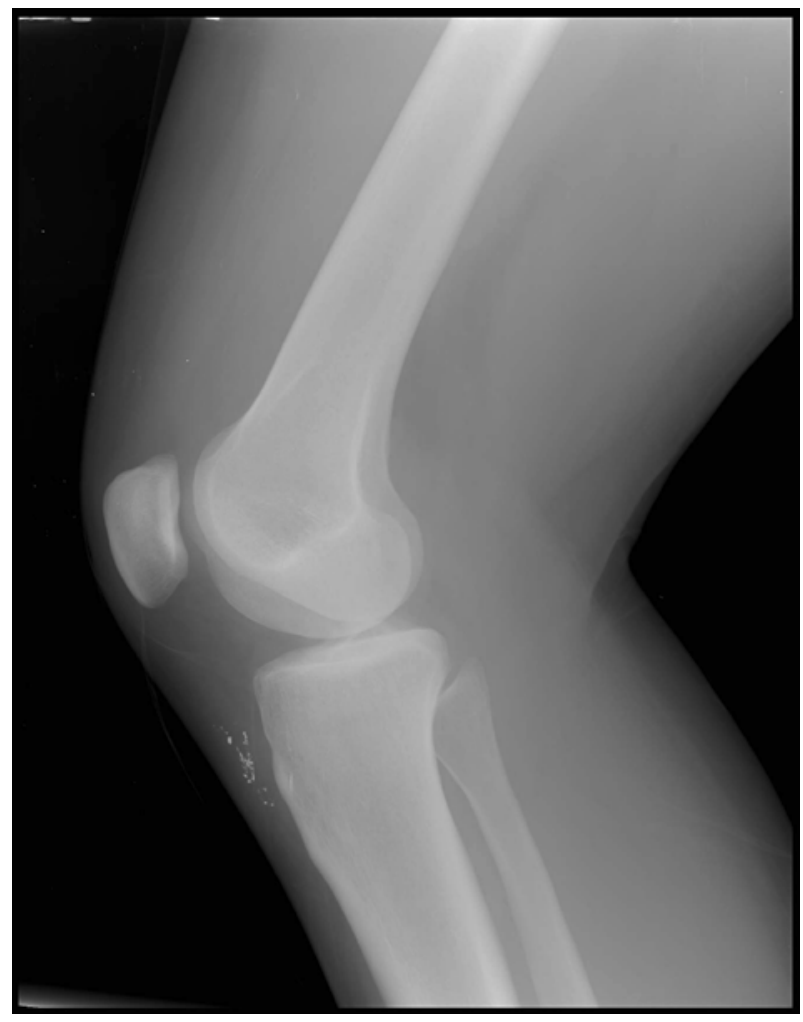




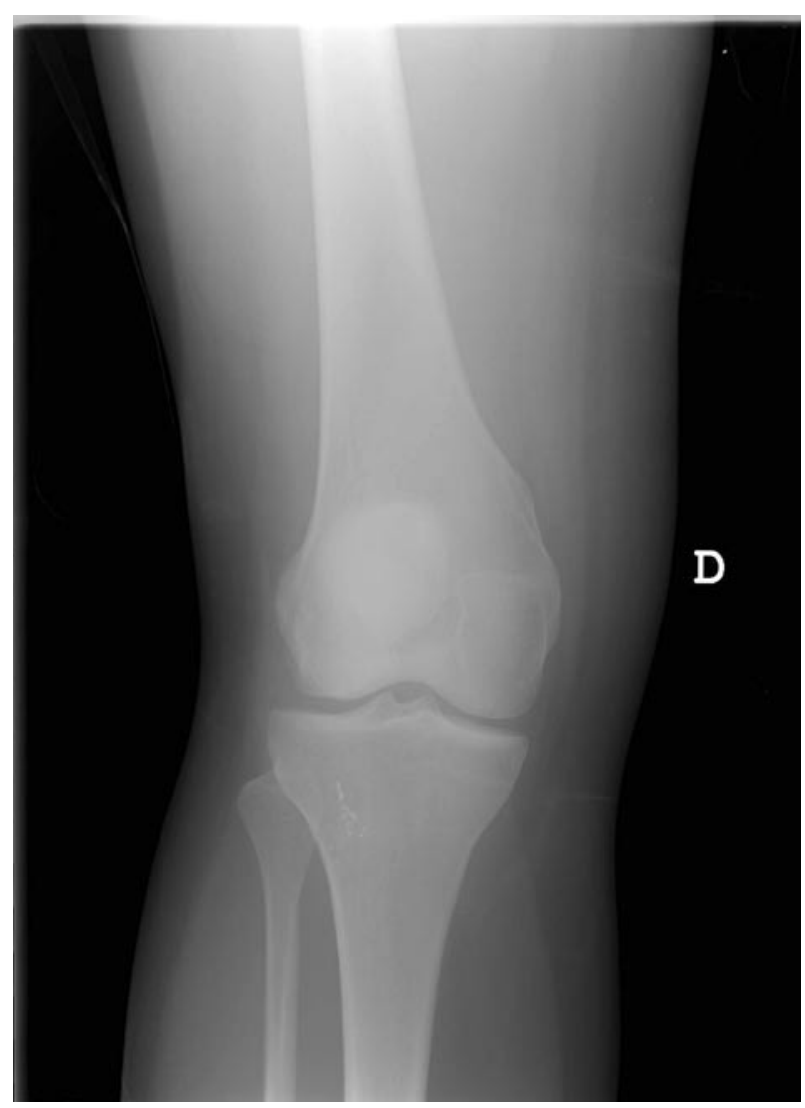

Tras el diagnóstico de bursitis química por mercurio, y teniendo en cuenta la toxicidad del metal, se procedió a la extracción de la bursa rotuliana en quirófano de forma urgente bajo escopia, para evitar que quedaran restos de mercurio en la articulación de la trabajadora.

Actualmente, la auxiliar no presenta ninguna secuela y está recuperada totalmente de su lesión.

\section{DISCUSIÓN Y CONCLUSIONES}

Tras el caso clínico aportado anteriormente, consideramos necesario tratar varios apartados:

1. Conocer los efectos que se pueden producir en la salud de los trabajadores expuestos a mercurio.

2. Analizar el accidente de trabajo para poder implantar o mejorar medidas preventivas que eviten este tipo de accidentes.

3. Reflexionar sobre la relación beneficio-riesgo del uso del mercurio en el ámbito sanitario.

\section{Efectos en la salud de los trabajadores expuestos a mercurio}

Las principales vías de entrada del mercurio en el organismo son la vía inhalatoria y la vía digestiva. La penetración subcutánea también es posible, dependiendo de la solubilidad, la concentración y el estado de la piel, sin embargo, su tasa es baja ${ }^{1}$.

La toxicidad del mercurio puede afectar a múltiples órganos, creando diferentes tipos de gravedad. Los principales órganos diana del mercurio son: 
- Sistema respiratorio: los síntomas engloban desde una irritación pulmonar (neumonitis química) hasta un edema agudo de pulmón.

- Sistema nervioso: temblor, Parkinsonismo y, en estadíos finales, demencia y pérdida de memoria.

- Sistema renal: nefrosis por intoxicación aguda.

- Aparato digestivo: gingivitis, estomatitis ulcero-membranosa y otros desórdenes gastrointestinales.

Estos síntomas están relacionados fundamentalmente con la intoxicación mercurial por vía digestiva o inhalatoria. Sin embargo, en nuestro caso clínico en el que la absorción fue subcutánea, puede que el mercurio solo produzca una inflamación local, eczema alérgico de contacto o irritación de la piel. Secundariamente, es posible que provoque insuficiencia renal, alteraciones del aparato digestivo, alteraciones neuro-psiquiátricas o anemias, que pueden llegar a ser graves, si se produce embolización del mercurio a los tejidos $^{2,3}$.

\section{Investigación y análisis del accidente}

En este accidente laboral ocurrido, valoramos la consecución de varios factores:

- En cuanto a las condiciones del puesto de trabajo, la auxiliar de enfermería refirió sobrecarga de trabajo, lo que conllevó a realizar la tarea de forma rápida y con falta de atención.

- La causa del accidente fue que la trabajadora resbaló, pero desconocemos si fue porque el suelo se encontraba en mal estado, por el calzado de la trabajadora o bien, por la falta de atención referida anteriormente.

- La caja de termómetros que transportaba, no estaba sellada ni cerrada de forma correcta, lo cual produjo la salida y rotura del los termómetros de su envase.

- La trabajadora no llevaba guantes ni otro equipo de protección que permitieran la manipulación del agente químico, en este caso, el mercurio.

- Tras el accidente, la trabajadora refirió no haberse cambiado de ropa de trabajo y no realizar ninguna medida de higiene.

- El mercurio no fue recogido del suelo ni se realizó ninguna medida de actuación frente al derrame.

\section{Medidas de actuación preventiva propuestas}

- Medidas organizacionales: muchos de los accidentes de trabajo se relacionan con la sobrecarga de trabajo, el estrés, la carga física o mental. Estos factores pueden condicionar la aparición de fatiga o falta de atención entre otros. Una buena organización en el equipo de trabajo, limitando las funciones, podrían ayudar a disminuir este tipo de accidentes.

- Mejoras en las instalaciones del puesto de trabajo: como comentábamos anteriormente, desconocemos si el suelo en el que resbaló la trabajadora estaba en malas condiciones, pero no está de más recordar que una mejora y/o mantenimiento adecuado de las instalaciones, también podrían favorecer a que estos accidentes fueron menos frecuentes.

- Medidas preventivas frente a agentes químicos:

- Lo más importante es hacer todo lo posible por sustituir el mercurio por otras sustancias que entrañen menos riesgos, y si ésto no fuera posible, adoptar todas las medidas de seguridad necesarias.

- Equiparse con equipos de protección individual. Quizá en este caso en el que la trabajadora transportaba, y no manipulaba el mercurio, los guantes y la ropa de trabajo adecuados, hubieran sido suficientes. 
- El mercurio, como agente químico, debe manipularse en sistemas herméticamente cerrados, para evitar, como en este caso, derrames tras la rotura de los termómetros.

- La cantidad de mercurio que sale de un típico termómetro roto es considerado un derramamiento pequeño. Las precauciones que deben tomarse ante un derrame pequeño son:

- No tocar el mercurio. Quitarse el reloj, ya que el metal puede quedarse adherido y ponerse guantes de goma. Usar foco de luz para localizar el mercurio.

- Las cantidades pequeñas de mercurio se pueden recoger con cinta adhesiva o con un gotero, cercando el área para evitar expandir el mercurio, guardándose posteriormente en un envase de plástico sellado.

- La ropa de trabajo contaminada y el mercurio recogido pueden desecharse en la basura regular, pero manteniéndola fuera en un lugar seguro, hasta su recogida.

- Las superficies deben limpiarse con fosfato de trisodium y agua. No utilizar aspiradora para la recogida del mercurio, ya que puede volatilizar los vapores del mercurio y promover su inhalación.

- Las ventanas/puertas en el área del derrame deben abrirse para ventilar la zona y las personas no relacionadas directamente con el derrame deben irse del área.

- Adoptar medidas de higiene estrictas.

- Informar y formar a los trabajadores sobre los riesgos que entraña el metal, ya que, la trabajadora refirió que la falta de conocimiento y la ignorancia al riesgo químico del mercurio, contribuyeron a que no tuviera cautela mientras transportaba los termómetros ${ }^{4,5}$.

\section{Uso del mercurio en el ámbito sanitario}

Como comentábamos en el apartado anterior, la primera medida de prevención ante todo riesgo es eliminarlo, en este caso, eliminar el mercurio de los aparatos de medición de uso hospitalario.

La Unión Europea en 2005, estableció una estrategia comunitaria sobre el mercurio, en la que se consideraba necesario introducir restricciones a la comercialización de determinados equipos de medición y control que contienen mercurio, reportando así beneficios para el medio ambiente y, a largo plazo, para la salud humana, al evitar el mercurio en los residuos.

A tal efecto, se aprobó a nivel europeo la Directiva 76/769/CEE del Consejo, de la que derivó el Real Decreto 1406/19889 que estableció una serie de limitaciones a la comercialización y al uso de ciertas sustancias y preparados peligrosos (dispositivos de medición que contiene mercurio).

Posteriormente la Orden PRE/222/2009 modificó, mediante el anexo I, dicho Real Decreto. En esta orden, se aprobaba que por motivos de viabilidad técnica y económica, sólo se prohibiría el uso del mercurio en los termómetros de uso general y sanitario, sin embargo, los aparatos de medición con una antigüedad de 50 años (antigüedades o bienes culturales), o aquellos aparatos de medición (que no sean termómetros) tales como esfingomanómetros, y siempre que sean para uso sanitario y no para el público general, serían eximidos de esta orden.

Sin embargo, se piensa que en 2011, la Comisión Europea prohibirá la venta de mercurio a la Unión Europea, lo que permitirá que, a la larga, se elimine el riesgo de exposición a mercurio, y por tanto, su toxicidad ${ }^{6,7}$. 


\section{BIBLIOGRAFÍA}

1. Gochfeld M. Cases of mercury exposure, bioavailability, and absorption. Ecotoxicology and Environmental Safety 2003; 56 (1): 174-179.

2. Tchounwou PB, Ayensu WK, Ninashvili N y Sutton D. Review: Environmental exposure to mercury and its toxicopathologic implications for public health. Environmental Toxicology 2003; 18 (3): 149-175.

3. Agency for Toxic Substances and Disease Registry (ATSDR). Toxicological Profile for Mercury. Atlanta, GA: U.S. Department of Health and Human Services, Public Health Service, 1999.

4. Guía técnica para la evaluación y prevención de los riesgos presentes en los lugares de trabajo relacionados con Agentes Químicos. Instituto Nacional de Seguridad e Higiene en el Trabajo. Madrid, 2003.

5. NTP 229: Mercurio inorgánico y metálico: protocolo de vigilancia médica. Instituto Nacional de Seguridad e Higiene en el Trabajo. Madrid.

6. Real Decreto 1406/1989 de 10 de noviembre, por el que se imponen limitaciones a la comercialización y al uso de ciertas sustancias y preparados peligrosos. BOE número 278 de 20/11/1989.

7. Orden PRE/222/2009, de 6 de febrero, por la que se modifica el anexo I del Real Decreto 1406/1989, de 10 de noviembre, por el que se imponen limitaciones a la comercialización y al uso de ciertas sustancias y preparados peligrosos (dispositivos de medición que contienen mercurio). BOE número 37 de 12/2/2009.

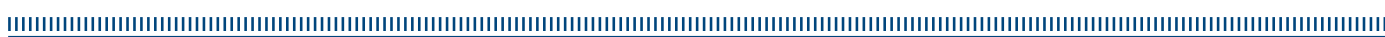

